\title{
Acoustics and Surface Pressure Measurements from Tandem Cylinder Configurations
}

\author{
Florence V. Hutcheson ${ }^{*}$, Thomas F. Brooks”, David P. Lockard^, Meelan M. Choudhari^ \\ NASA Langley Research Center, Hampton, Virginia \\ Daniel J. Stead** \\ Northrop Grumman, Hampton, Virginia
}

\begin{abstract}
Acoustic and unsteady surface pressure measurements from two cylinders in tandem configurations were acquired to study the effect of spacing, surface trip and freestream velocity on the radiated noise. The Reynolds number ranged from $1.15 \times 10^{5}$ to $2.17 \times 10^{5}$, and the cylinder spacing varied between 1.435 and 3.7 cylinder diameters. The acoustic and surface pressure spectral characteristics associated with the different flow regimes produced by the cylinders' wake interference were identified. The dependence of the Strouhal number, peak Sound Pressure Level and spanwise coherence on cylinder spacing and flow velocity was examined. Directivity measurements were performed to determine how well the dipole assumption for the radiation of vortex shedding noise holds for the largest and smallest cylinder spacing tested.
\end{abstract}

\section{Introduction}

During airport approach, the noise radiated from an aircraft landing gear is a dominant airframe noise source. A number of numerical ${ }^{1-7}$ and experimental studies ${ }^{8-11}$ have been conducted in order to identify and model the noise generation mechanisms from landing gear configurations. Tandem Cylinders have been studied because they can model a range of component level interactions. Since many components of a landing gear (the struts, the cables, the axles) can be modeled by rods of various lengths and cross-sections, understanding the generation mechanisms of the noise radiated from single and multiple rod configurations ${ }^{12,13}$ is of relevance to the landing gear noise reduction effort.

In the present study, unsteady surface pressure and acoustic measurements were obtained for two cylinders in tandem configurations to investigate the effect of cylinder spacing, surface trip and flow speed on the radiated noise and cylinder's surface pressure. An additional test objective was to establish an experimental acoustic and unsteady surface pressure data set for assessment of simulation-based prediction tools ${ }^{14-16}$. The results from this experimental study also complement a set of flow visualization, Particle Image Velocimetry and hot-wire anemometry data ${ }^{17,}{ }^{18}$ acquired for a subset of the tandem cylinder configurations presented in this paper.

\section{Test description}

This experiment was performed at NASA Langley Research Center in the Quiet Flow Facility (QFF). The QFF is an open jet facility equipped with a 2 by 3 foot rectangular open jet nozzle.

\footnotetext{
*Senior Research Engineer, Aeroacoustics Branch, Senior Member AIAA

\# Senior Research Scientist, Aeroacoustics Branch, Fellow AIAA

${ }^{\wedge}$ Senior Research Engineer, Computational Aerosciences Branch, Senior Member AIAA

*** Senior Engineer
} 


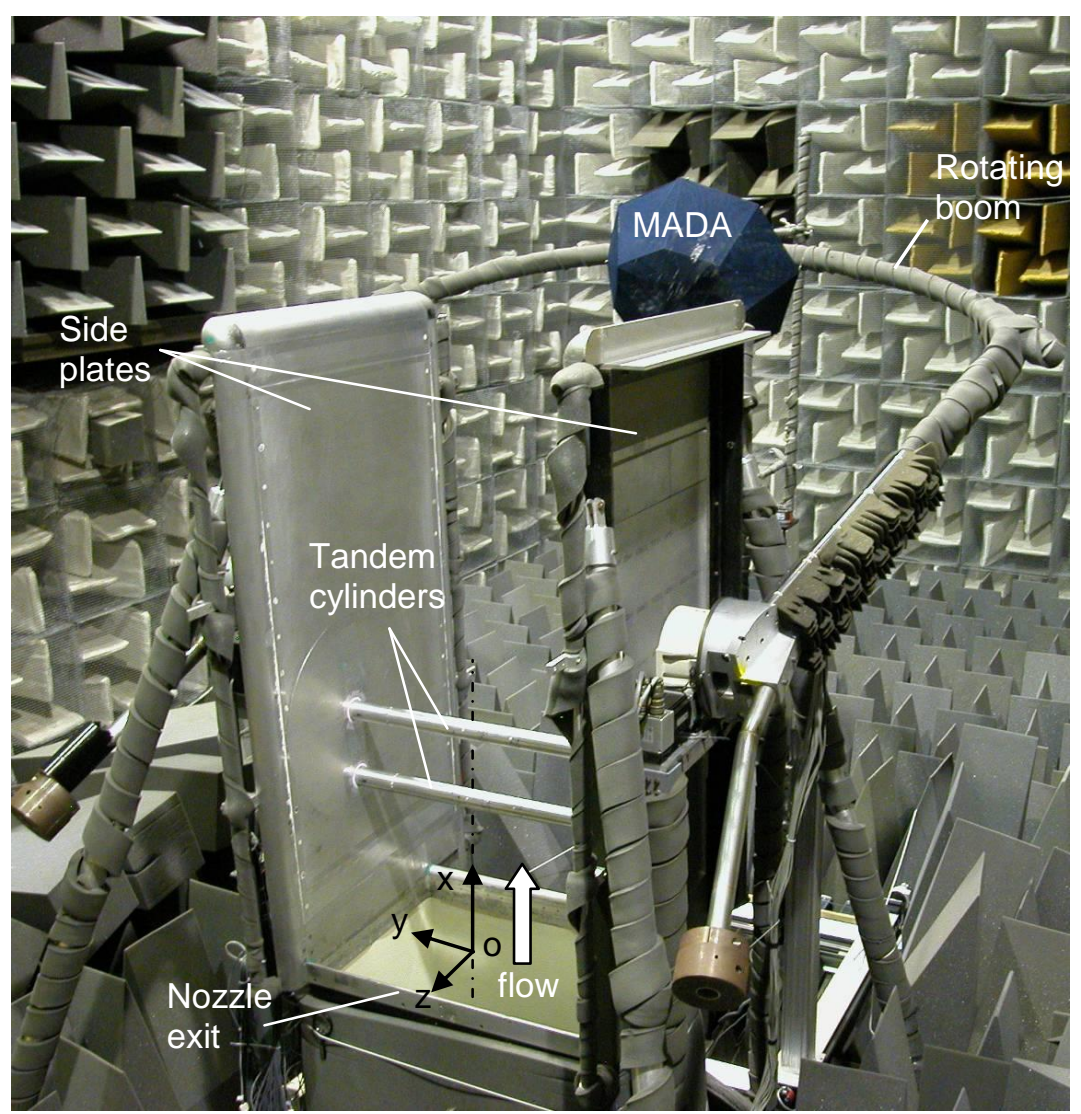

Figure 1. Quiet Flow Facility.

The test model consisted of two identical cylinders that were 3 foot in span and 2.2 inches in diameter. The cylinders were supported above the nozzle by two vertical side plates that are mounted to the short sides of the nozzle (see Figure 1). The cylinders were aligned streamwise with a separation distance (center to center), $\mathrm{L}$, ranging from $1.435 \mathrm{D}$ to $3.7 \mathrm{D}$, where $\mathrm{D}$ is the cylinder diameter. The cylinders were located well within the smooth core flow. The upstream cylinder was positioned at a fixed location on the nozzle centerline, 19 inches above the nozzle exit plane (referring to Figure 1, the center of the cylinder at midspan was positioned at $\mathrm{x}=19$ inches and $\mathrm{y}=\mathrm{z}=0$ ). The upstream cylinder remained fixed while the downstream cylinder was moved along the nozzle center line to adjust the separation distance. The cylinders were tested tripped and un-tripped. For the tripped model configurations, a strip of 0.005 inches thick, serrated aluminum tape was placed between azimuthal locations of $50^{\circ}$ and $60^{\circ}$ from either side of the leading stagnation point. Both cylinders were instrumented with unsteady surface pressure transducers (Figure 2). The transducers were distributed on a spanwise row at an azimuthal location of $135^{\circ}$. The spanwise row covered the center portion of each cylinder over a span of $7 \mathrm{D}$ (15.75 inches). Transducers were also installed with $45^{\circ}$ azimuthal spacing around the circumference of each cylinder, at mid-span. 


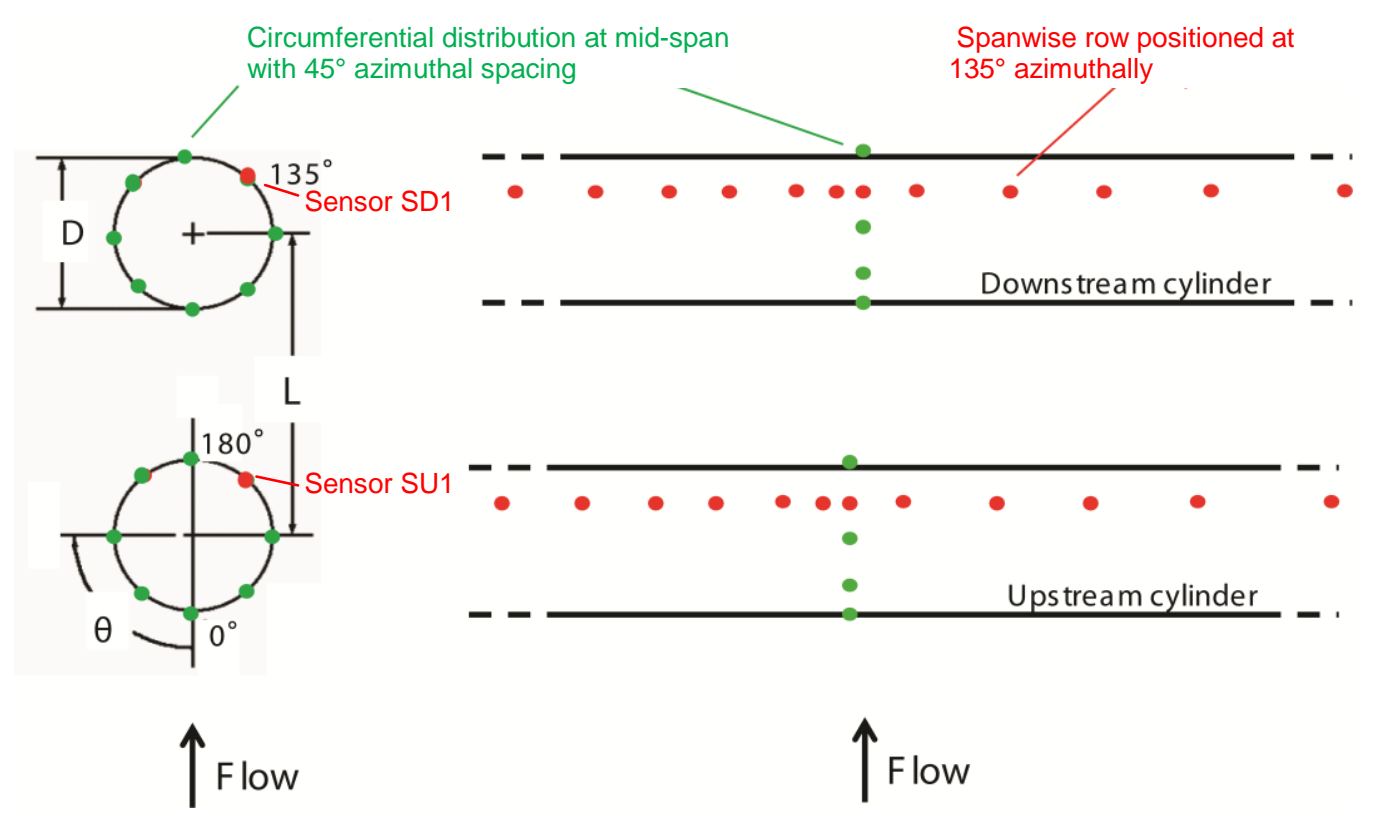

Figure 2. Schematic of unsteady surface pressure sensors layout. Sensor location: • (spanwise row), $\bullet$ (mid-span ring)

Noise and surface pressure measurements were acquired at flow Mach number ranging from 0.09 to 0.17. A sketch of the acoustic test setup is shown in Figure 3. Three microphones were positioned on one side of the test section. These microphones were oriented at a point, $\mathrm{C}(39.5,0,-5.4)$, located 5.4 inches off the nozzle centerline and 39.5 inches above the nozzle exit plane. (This particular orientation of the microphones was chosen for convenience in the setup of the test hardware.) These three microphones were positioned 78.5 inches away from that center point and at, respectively, $\Phi=60^{\circ}, 90^{\circ}$ and $120^{\circ}$ elevation. For a subset of the tandem cylinders configurations tested, a Medium Aperture Directional Array (MADA) was used to make noise measurements at intermediate elevation angles (namely, $45^{\circ}, 75^{\circ}, 105^{\circ}$ and $135^{\circ}$ ). The MADA was positioned 60 inches from point $C$.

Transient data recorders controlled by a workstation were used to acquire the data from all microphone and surface pressure transducer channels simultaneously at a sampling rate of $25.6 \mathrm{kHz}$. High pass and low pass filters, set respectively at $5 \mathrm{~Hz}$ and $10 \mathrm{kHz}$, were used to condition the outputs from each channel. The noise spectra were obtained by partitioning each time signal into 1000 non-overlapping segments of $2^{13}$ samples, and each time history segment was Fourier transformed using a Hamming window for signal conditioning. The resulting frequency resolution was $3.125 \mathrm{~Hz}$. The background noise pressure field (measured for each of the flow Mach numbers tested, with air flowing through the empty test section) was subtracted (on a $\mathrm{p}^{2}$ basis) from the noise pressure field measurements obtained (at the same gain setting) from the different tandem cylinders configurations. 


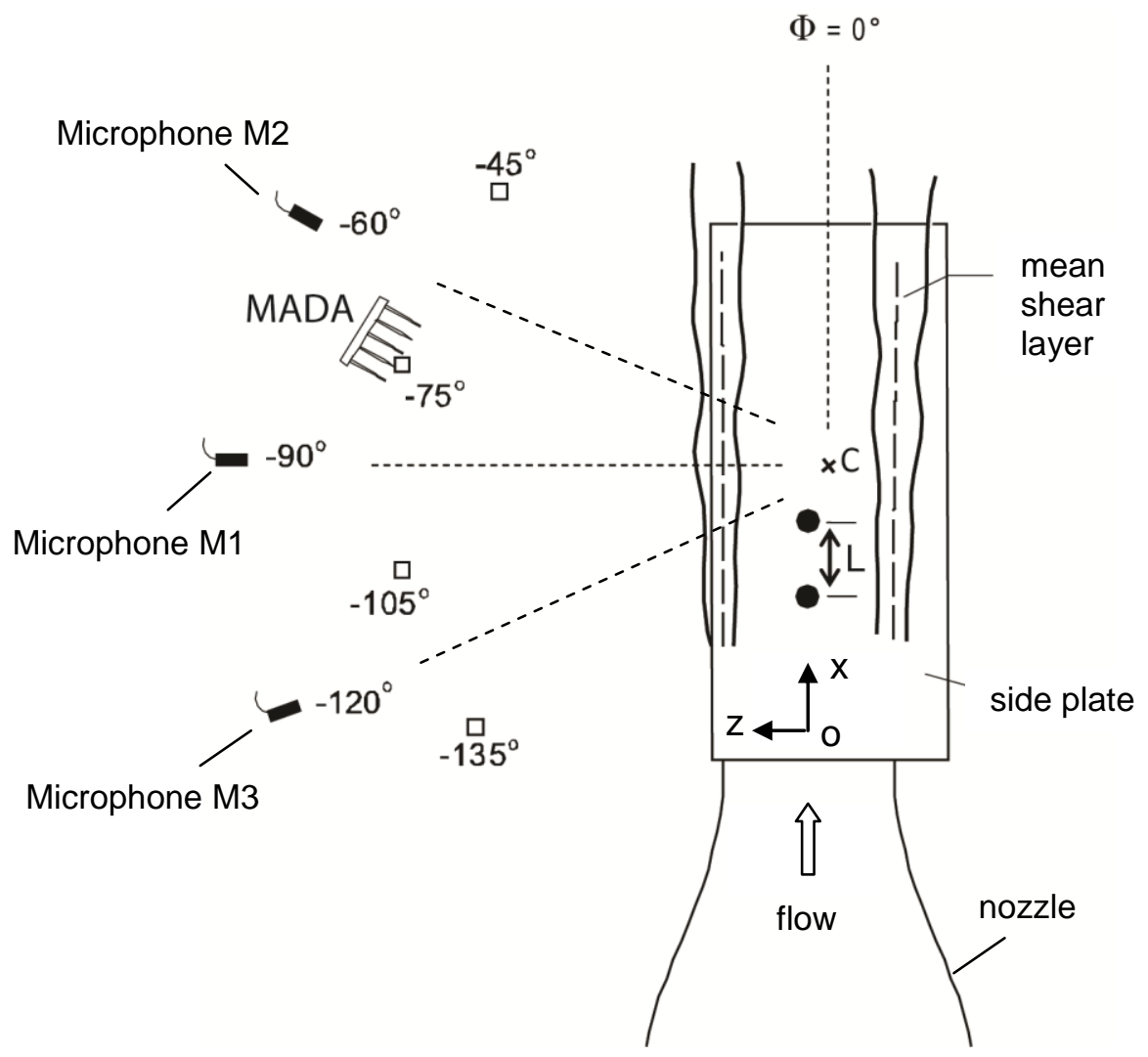

Figure 3. Sketch of acoustic setup.

\section{Background and theory}

\subsection{Flow regimes and state of flow}

Measurements were performed for the following cylinder spacings $\mathrm{L} / \mathrm{D}=1.435, \mathrm{~L} / \mathrm{D}=2, \mathrm{~L} / \mathrm{D}=2.5, \mathrm{~L} / \mathrm{D}=3$, $\mathrm{L} / \mathrm{D}=3.4$, and $\mathrm{L} / \mathrm{D}=3.7$. Each of these spacings is expected to be associated with a specific flow regime. Zdravkovich has categorized the different possible flow regimes associated with wake interference for increasing $\mathrm{L} / \mathrm{D}$ as follows ${ }^{19}$ : (i) the free shear layers separated from the upstream rod do not reattach onto the downstream rod. The vortex street behind the downstream rod is formed by the free shear layers separated from the upstream rod; (ii) the free shear layers separated from the upstream rod may reattach alternately, permanently or intermittently onto the downstream rod. Vortex shedding takes place only behind the downstream rod; (iii) vortex shedding occurs intermittently behind the upstream rod; (iv) the eddies from the upstream rod pair with the eddies behind the downstream rod. The two vortex streets are synchronized in phase and frequency; (v) uncoupled vortex shedding takes place behind both rods. These different flow regimes are summarized and illustrated in Table 1.

For the Reynolds number range considered in this study (namely, $1.15 \times 10^{5}$ to $2.17 \times 10^{5}$ ), without surface trip, the state of flow ${ }^{19}$ for a single cylinder is expected to remain subcritical (laminar boundary layer with transition to turbulence occurring in the free shear layers). A turbulent boundary layer (generally associated with higher Reynolds numbers) can however be achieved with the application of surface trip (transition strips), or can sometime be instigated by freestream turbulence ${ }^{12,19}$. For a tandem cylinder configuration, in the absence of surface trip, the subcritical state of flow is expected to apply only to the upstream cylinder. The downstream cylinder is immersed in the turbulent wake of the upstream cylinder which is expected to induce transition to turbulence in the boundary layer. This will be verified from the results presented in section 4.1. 
Table 1. Tandem rods - Interference flow regimes (illustration similar to that in Reference 19).

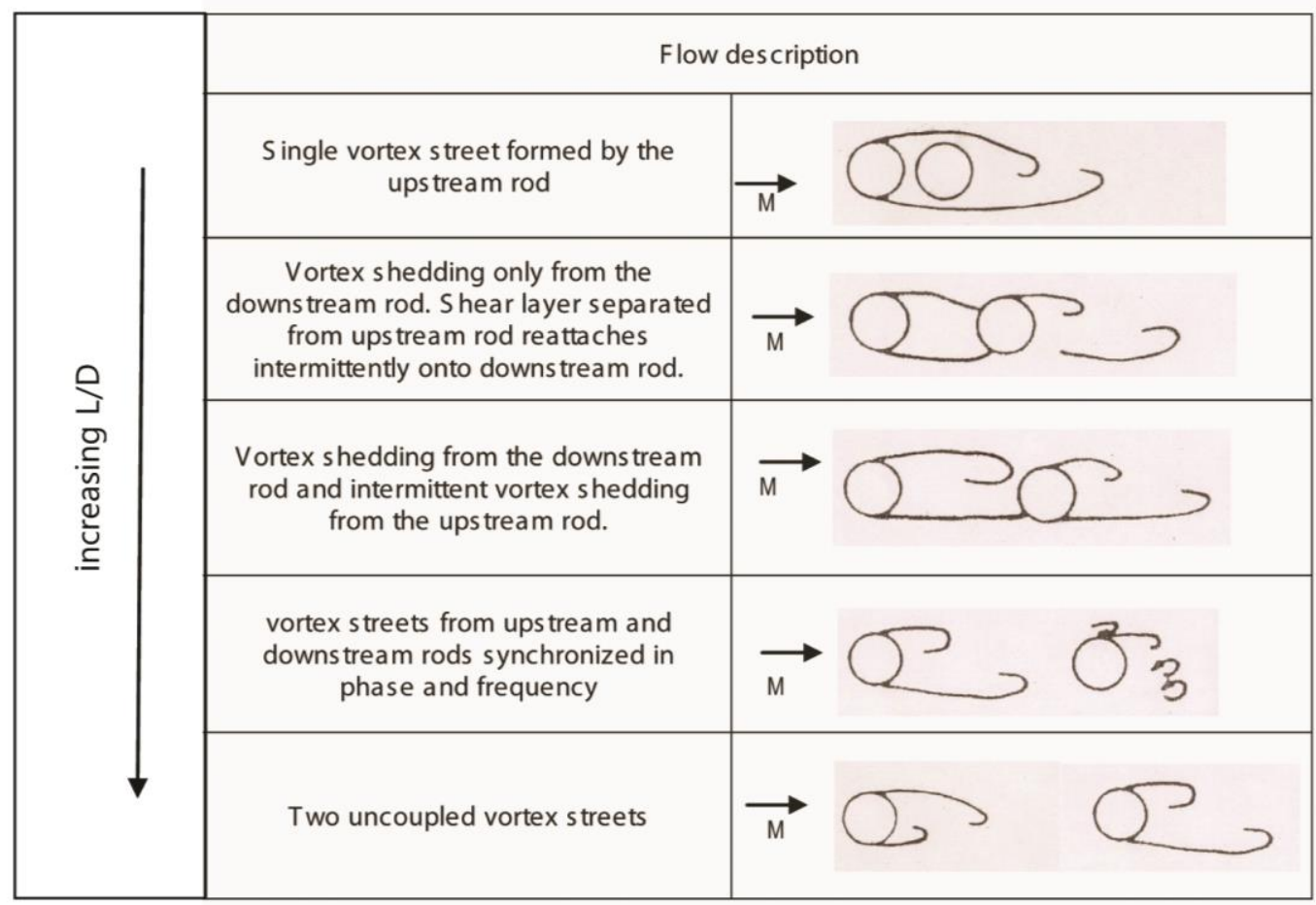

\subsection{Predictions}

In the range of Reynolds number over which periodic vortex shedding takes place, the noise is tone-like and radiates like a dipole. The following model has been developed and most commonly used ${ }^{20,21}$ to predict the mean square acoustic pressure of the noise field

$$
p^{2}(r)=\frac{\rho^{2} c^{4} M^{6} L D L_{c} S_{t}^{2} C_{L}^{2}}{16 r^{2}} D_{r}\left(\phi_{e}\right) .
$$

In Eq.(1), $\rho$ is the air density, $c$ is the speed of sound, $M$ is the flow Mach number, $L$ is the rod span, $D$ is the rod diameter, $L_{c}$ is the spanwise correlation length of the surface pressure fluctuation normalized by $D$, $\mathrm{S}_{\mathrm{t}}$ is the Strouhal number, $\mathrm{C}_{\mathrm{L}}{ }^{2}$ is the mean-square coefficient of the fluctuating lift, $\mathrm{r}$ is the distance from the rod to the observer and $\mathrm{D}_{\mathrm{r}}\left(\phi_{\mathrm{e}}\right)$ is the directivity function for the radiated noise. The Strouhal number is defined as

$$
S_{t}=\frac{f_{\text {peak }} D}{U}
$$

where $U$ is the freestream velocity and $f_{\text {peak }}$ is the spectral peak frequency. $D_{r}\left(\phi_{e}\right)$ can be modeled as the directivity function for a translating dipole ${ }^{22}$.

$$
D_{r}\left(\phi_{e}\right)=\frac{\sin ^{2} \phi_{e}}{\left(1+M \cos \phi_{e}\right)^{4}}
$$

where $\Phi_{\mathrm{e}}$ is the angle between the freestream and the observer, and is expressed in the retarded emission coordinates system ${ }^{23}, 24$. Equations (1), (2) and (3) will be used to scale some of the results presented in section 4 to study the dependence of the vortex shedding noise Sound Pressure Level (SPL) with respect to known parameters, and to determine how well these equations model the radiation of the vortex shedding noise for some of the tandem cylinder configurations tested. 


\subsection{Spanwise coherence}

The spanwise variation of the coherence $\gamma^{2}$ of the surface pressure along each cylinder was also examined. This spanwise coherence was calculated for each model configuration by using an energy weighted average of the coherence over a 13-bin frequency band centered at the spectral peak frequency. The coherence is defined as

$$
\gamma_{i}^{2}\left(f_{\text {peak }}\right)=\frac{G_{1, i}{ }^{2}\left(f_{\text {peak }}\right)}{G_{1,1}\left(f_{\text {peak }}\right) G_{i, i}\left(f_{\text {peak }}\right)}, i=1, \ldots N
$$

where $\mathrm{N}$ is the number of surface pressure sensors distributed along the span of each cylinder, and $\mathrm{G}_{1,1}(\mathrm{f})$, $\mathrm{G}_{1, \mathrm{i}}(\mathrm{f})$ and $\mathrm{G}_{\mathrm{iil}}(\mathrm{f})$ are the cross spectra and auto spectra of the pressure sensors signals. In the remainder of this paper, the spanwise correlation length $\mathrm{L}_{\mathrm{c}}$ will be defined as the span (divided by $\mathrm{D}$ ) over which the coherence factor, $\sqrt{\gamma^{2}}$, is greater or equal to 0.5 . This definition of $L_{c}$ is suitable for the purpose of the present analysis but was chosen somewhat arbitrarily. A more accurate definition of $\mathrm{L}_{c}$ would be $\mathrm{e}^{25}$ $\mathrm{L}_{\mathrm{c}}=\frac{1}{D} \int \sqrt{\gamma^{2}} \eta d \eta / \int \sqrt{\gamma^{2}} d \eta$ where $\eta$ is defined by the spacing of the pressure sensors along the cylinder span.

\section{Experimental results}

The results from this tandem cylinder experiment are presented next. The noise spectra shown in sections 4.1 through 4.3 were obtained from measurements acquired with microphone M1 (see Fig. 3), while the unsteady surface pressure spectra shown for the upstream and downstream cylinders were obtained from sensors SU1 and SD1, respectively (see Fig. 2). Although data is available up to $10 \mathrm{kHz}$, the spectra shown are limited to a lower frequency range, as all relevant noise and surface pressure spectral characteristics observed were located below $1 \mathrm{kHz}$.

\subsection{Effect of surface trip}

The tandem cylinders were tested tripped and un-tripped. The surface trip was used to force the boundary layer to a turbulent state, simulating higher Reynolds number flows. The noise spectra obtained for the different tandem cylinder configurations are shown in Figure 4. It is seen that tripping both cylinders compared to tripping only the upstream cylinder offers little difference in the radiated noise spectra. This was observed for all L/D values and flow speeds tested. The corresponding unsteady surface pressure spectra are shown in Figure 5. As with the noise spectra (except for L/D of 3.7), it is seen that tripping only the upstream cylinder compared to tripping both cylinders results in little difference in the upstream or downstream unsteady surface pressure. This was observed at all flow speeds. These results are consistent with the wake from the upstream cylinder successfully instigating transition in the boundary layer of the downstream cylinder. Only for L/D of 3.7 did the difference in tripping lead to slightly different surface pressure levels on the downstream cylinder. However, this difference in surface pressure levels (which could be related to a possible start of change in flow regime) was seen only for frequencies below $5 \mathrm{kHz}$ and it did not affect the noise spectra (as is seen in Figure 4).

It is further seen from the noise spectra that the absence of surface trip led to higher peak SPL and broadband noise levels (this was observed at all speeds except for $\mathrm{L} / \mathrm{D}=3$, at the lowest flow Mach number tested). The increase in broadband noise levels for the un-tripped configurations ranged approximately from $0.5 \mathrm{~dB}$ to $8 \mathrm{~dB}$. No trend or correlation could be established between the level of increase in broadband noise and changes in flow speed or cylinder spacing. It is believed that the higher broadband noise levels are associated with the more pronounced laminar instabilities and irregularities in the surface pressure and thus in the acoustic pressure radiated from the un-tripped model configurations. These instabilities in the time signal (as those shown in Figure 6) result in more energy being spread out over a broad frequency range in the noise spectra.

Since nearly identical results were obtained for the two different surface trip configurations, the results obtained with only one of these configurations (upstream cylinder tripped) will be presented in the remainder of this paper (i.e., the results presented for the tripped configurations will correspond to the results obtained when only the upstream cylinder is tripped). 

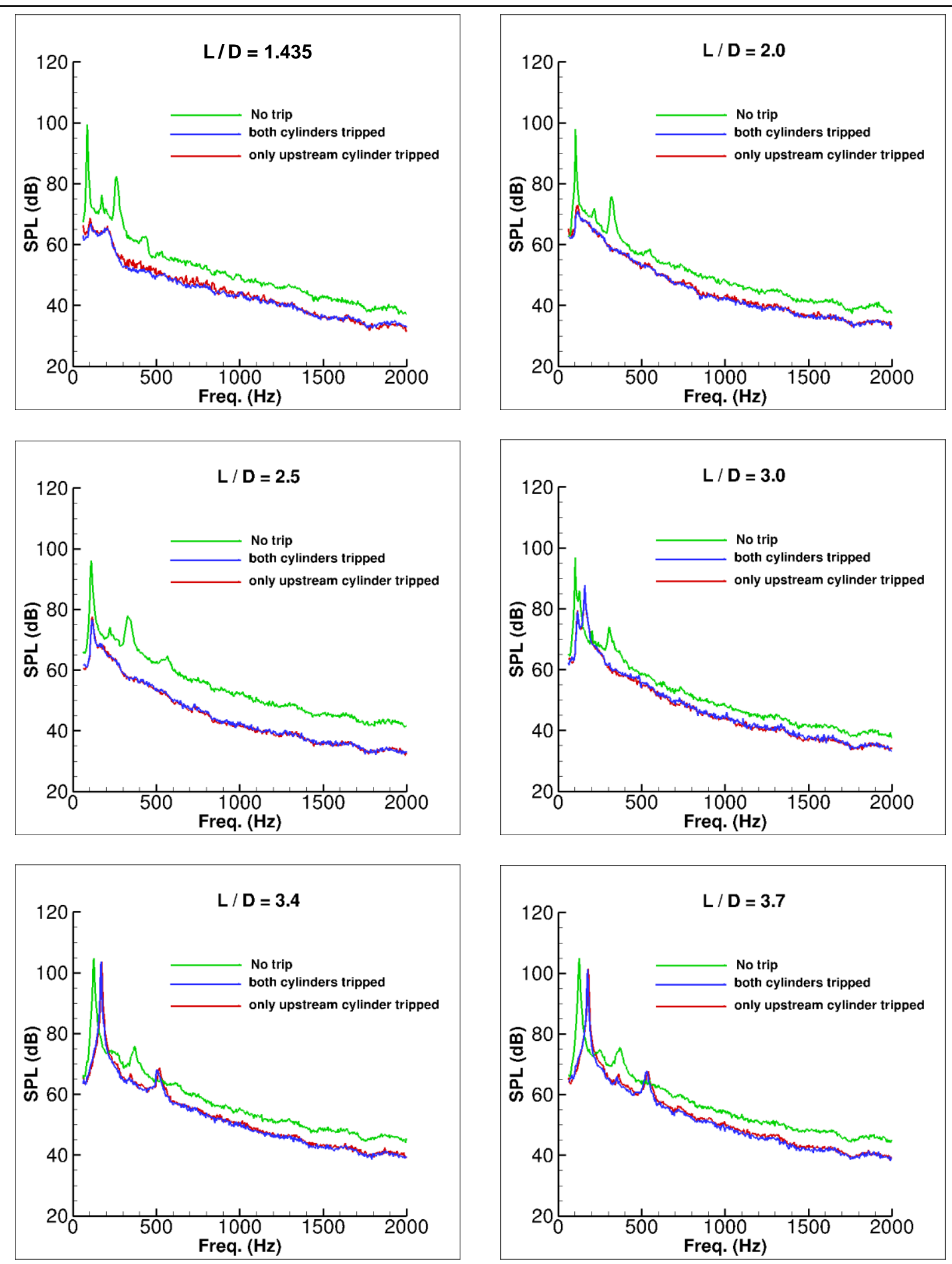

Figure 4. Effect of surface trip on noise autospectra. Data from microphone M1. Flow Mach number 0.128. 

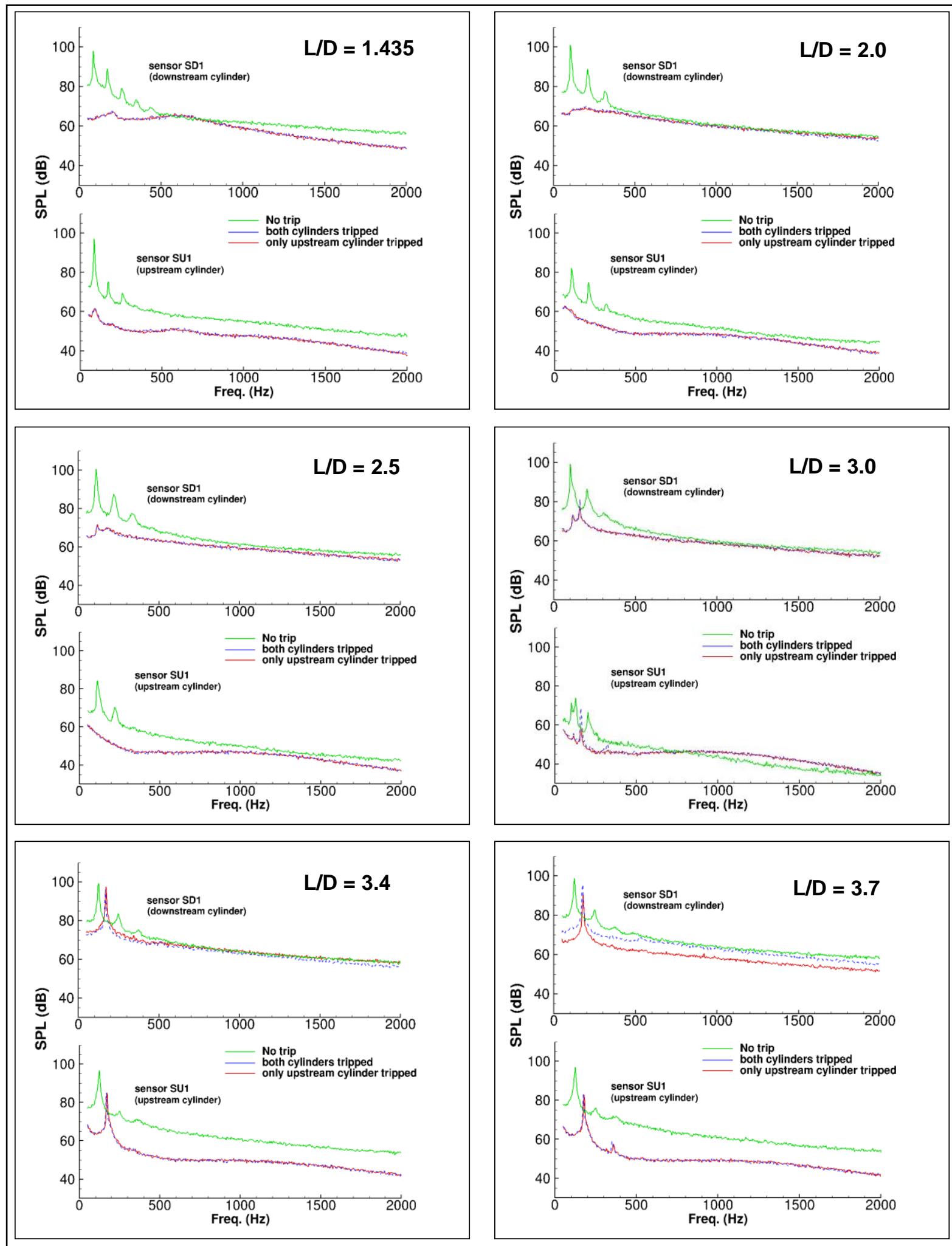

Figure 5. Effect of surface trip on unsteady surface pressure autospectra. Data from sensors SU1 and SD1 (see Figure 2 for sensor location). Flow Mach number 0.128. 


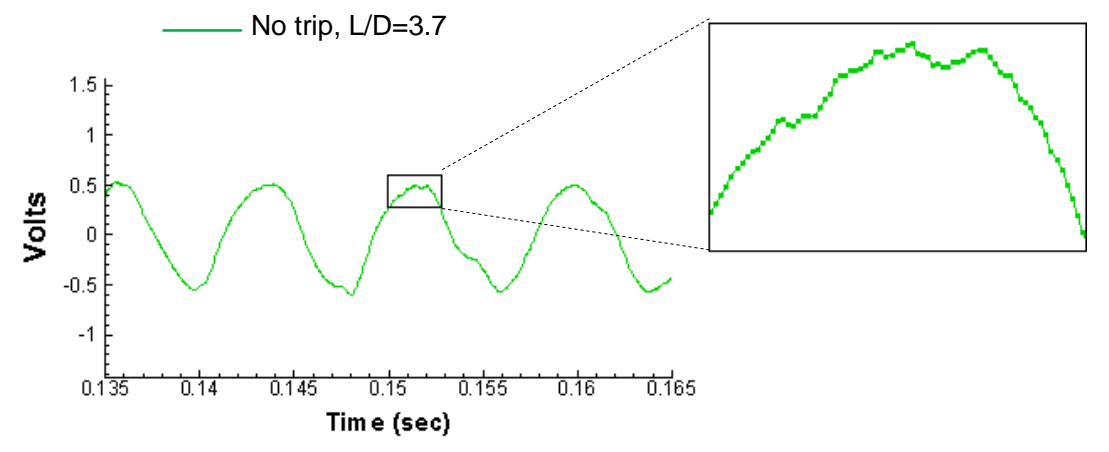

Figure 6. Acoustic signal (in voltage units) time history from Microphone M1. Mach = 0.128.

\subsection{Effect of cylinder spacing}

For tripped and untripped cylinders, the effect of cylinder spacing on the surface pressure spectra, noise spectra and spanwise coherence is discussed next. (In the figures presented in this section, the spectral levels of the unsteady surface pressure measurements shown were arbitrarily lowered by $42 \mathrm{~dB}$ to facilitate comparison with the corresponding noise spectra.)

For the untripped cylinder configurations (and as noted in Figure 4), the noise spectra displayed large tones for all of the cylinder spacings examined. The large tones seen in each of the noise spectra were also seen in the unsteady surface pressure spectra from both cylinders. This is shown in Figure 7 for two L/D values. These tones indicate that vortex shedding takes place for each of these tandem cylinder configurations. The spanwise coherence of the unsteady surface pressure along each cylinder is shown in Figure 8. It is seen that, except for the narrowest spacing tested, the spanwise coherence and correlation length along the downstream cylinder were greater than for the upstream cylinder. For $2.0 \leq \mathrm{L} / \mathrm{D} \leq 3.7$, Lc values for the upstream cylinder remained between 2.5 and 4.5 , while Lc values were greater than 4 for the downstream cylinder. This difference in correlation length between upstream and downstream cylinders may be related to the difference in the state of the boundary layers on the two cylinders (laminar on one and turbulent on the other); the shedding from a turbulent boundary layer being expected to be more stable (i.e., less sensitive to small changes in flow or surface condition along the model span) than when the boundary layer is laminar. For the particular case of $\mathrm{L} / \mathrm{D}=1.435$, the coherence level along the upstream cylinder were the highest measured. Thus, for $\mathrm{M} \geq 0.11$, it remained above 0.85 over the portion of the cylinder span (7D) where surface pressure measurements were acquired. It will be seen further below that when a transition strip is applied to the upstream cylinder, this coherence level, correlation length, and associated tone weaken drastically.

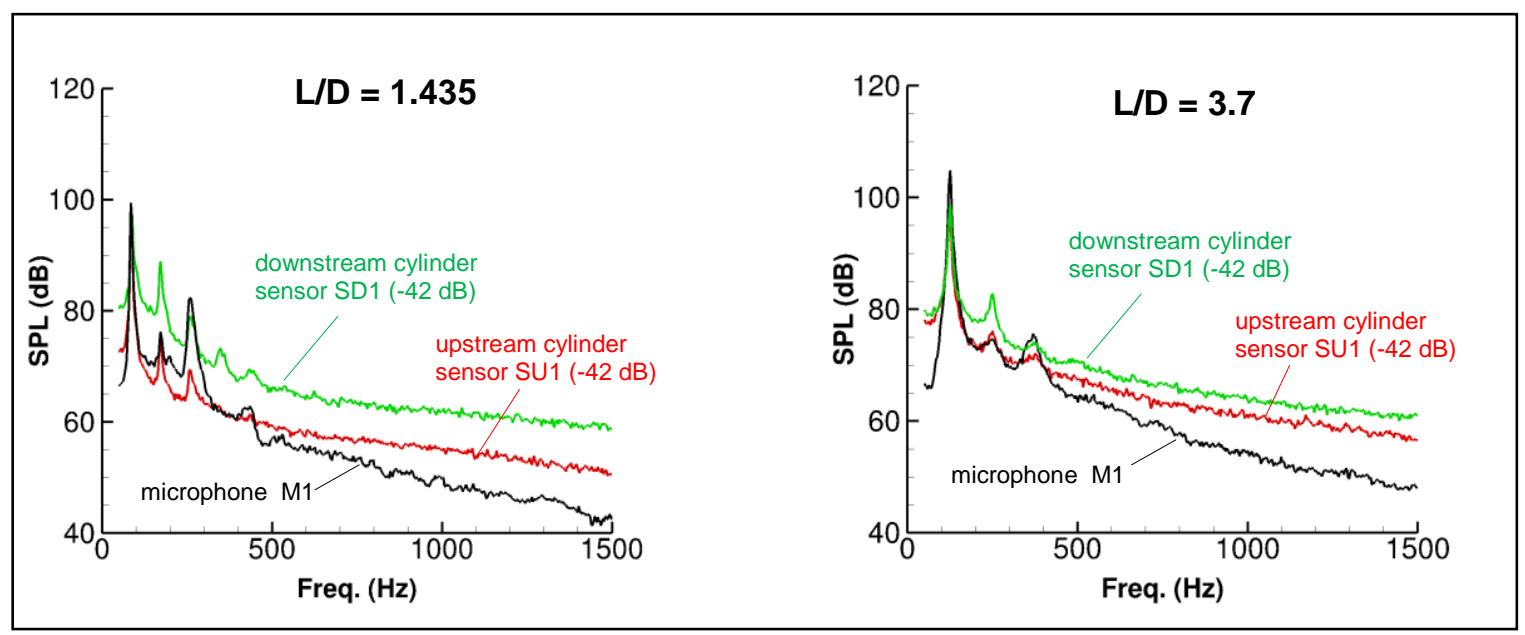

Figure 7. Comparison of radiated noise and surface pressure spectra. No surface trip. Mach $=0.128$. 


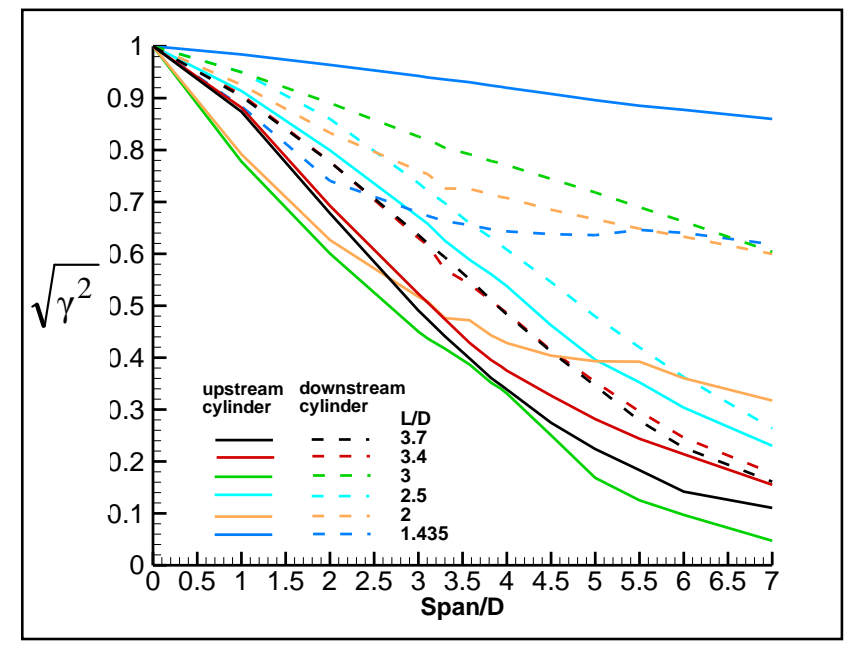

Figure 8. Coherence factor level of the unsteady surface pressure along span of upstream and downstream cylinders at the noise spectral peak frequency. No surface trip. Mach number 0.128 .

For the tripped cylinders, change in cylinder spacing was associated with clear changes in flow regime and in the noise spectral characteristics. The spanwise coherence, noise and surface pressure spectra associated with each L/D for the tripped cylinder configurations are examined next.

The noise and surface pressure spectra obtained for L/D of 3.7 and a Mach number of 0.128 are compared in Figure 9(a). The corresponding spanwise coherence of the surface pressure signals for each cylinder is shown in Figure 9(b). It is seen that the sharp tone in the noise spectrum associated with the vortex shedding is also present (at the same frequency) in the surface pressure spectra from each cylinder. The spanwise coherence plot also indicates that both cylinders contribute to the vortex shedding process with the same "efficiency". The same spanwise coherence and spectral characteristics were observed for L/D of 3.4 and 3.7 at all speeds, as well as for L/D of 3.0 at the two lowest speeds (namely, Mach 0.09 and 0.11 ). These characteristics are consistent with the $4^{\text {th }}$ flow regime listed in Table 1 (two vortex streets synchronized in frequency. Eddies from the upstream rod pair with the eddies behind the downstream rod).

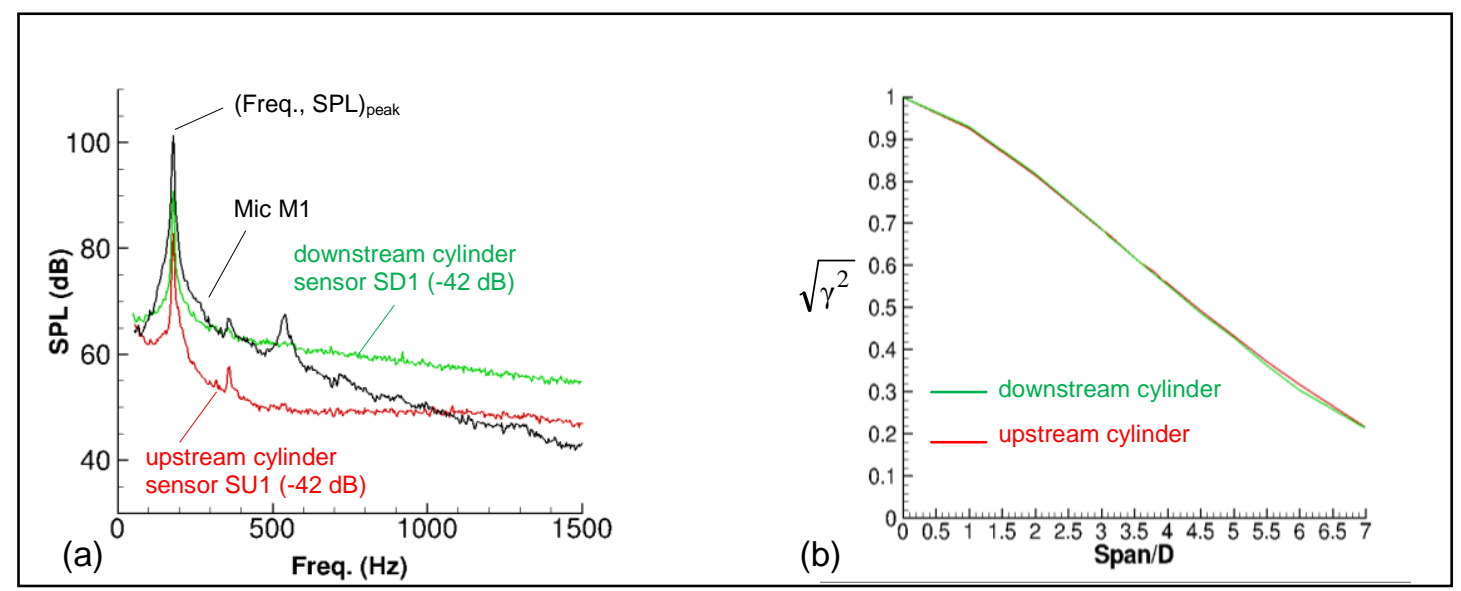

Figure 9. L/D=3.7; Flow Mach number 0.128; upstream cylinder tripped; (a) Noise and surface pressure spectra; (b) Spanwise coherence factor level at the noise spectral peak frequency.

The noise, surface pressure spectra and spanwise coherence obtained for $\mathrm{L} / \mathrm{D}$ of 2.5 and at Mach number of 0.128 are presented in Figure 10(a) and (b). It is seen that the main tone in the noise spectrum can only 
be found in the surface pressure spectrum from the downstream cylinder. The surface pressure spectrum from the upstream cylinder is broadband in nature, which indicates an absence of well established periodic phenomena (such as vortex shedding). The lack of spanwise coherence between pressure sensors signals along the upstream cylinder re-enforces that point. Similar spanwise coherence and spectral characteristics were observed for $\mathrm{L} / \mathrm{D}$ of 2.5 and 2.0 at all speeds, as well as for $\mathrm{L} / \mathrm{D}$ of 3.0 at the highest speeds tested (Mach 0.17). These characteristics are consistent with the $2^{\text {nd }}$ flow regime listed in Table 1 (vortex shedding only from the downstream cylinder). It is noted however that for $\mathrm{L} / \mathrm{D}$ of 2.0, the vortex shedding tone and correlation length on the downstream cylinder were significantly weaker than for L/D of 2.5.

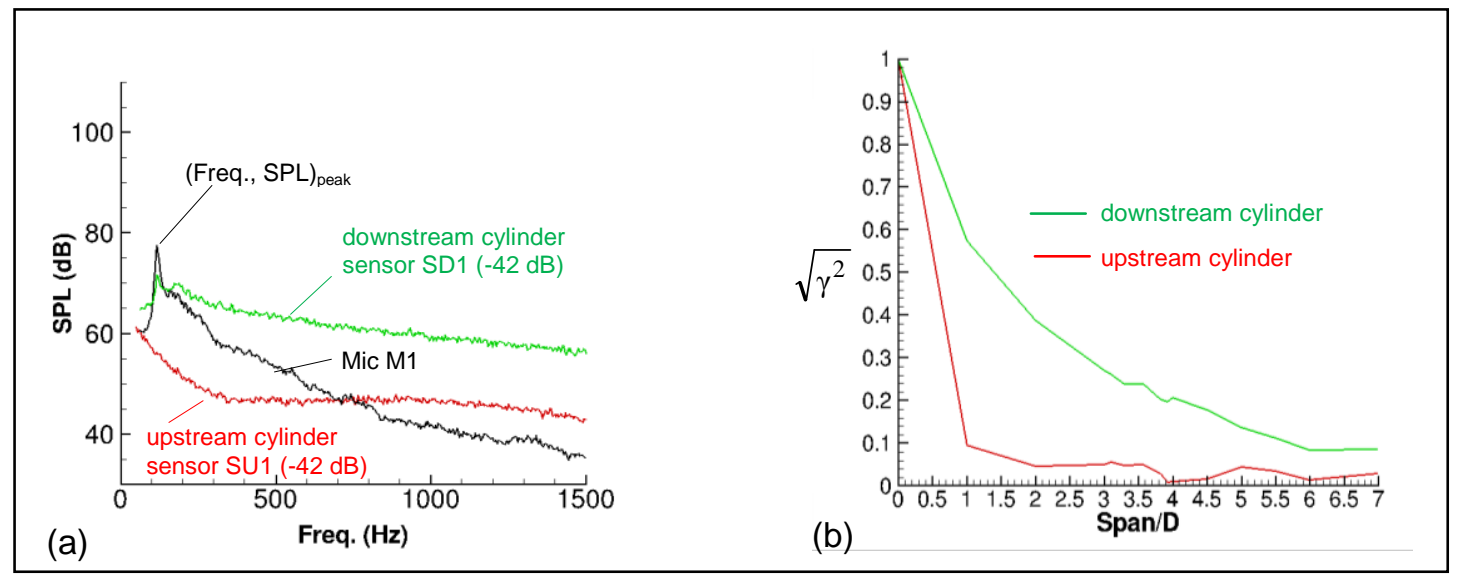

Figure 10. L/D=2.5; Flow Mach number 0.128; upstream cylinder tripped; (a) Noise and surface pressure spectra; (b) Spanwise coherence factor level at the noise spectral peak frequency.

The noise, surface pressure spectra and spanwise coherence obtained for L/D of 3.0 and a Mach number of 0.128 are presented in Figure 11(a) and (b). It is seen that the noise and surface pressure spectra each display two tones, the larger tone occurring at approximately the same spectral peak frequency as in the $\mathrm{L} / \mathrm{D}=3.7$ and 3.4 test cases, while the smaller tone has the same peak frequency as in the $\mathrm{L} / \mathrm{D}=2.5$ and 2.0 cases. At a flow Mach number of 0.128, the level of spanwise coherence for $L / D=3.0$ (Figure 11(b)) is also found to be lower than for the $\mathrm{L} / \mathrm{D}=3.7$ and 3.4 cases, but higher than for the $\mathrm{L} / \mathrm{D}=2.5$ and 2.0 cases. These results are consistent with a flow regime that is transitioning between the two flow regimes $\left(2^{\text {nd }}\right.$ and $4^{\text {th }}$ flow regime listed in Table 1) discussed above. Thus, as the flow Mach number increases, the flow regime for $\mathrm{L} / \mathrm{D}=3.0$ transitions from a "two synchronized vortex streets" regime to a "downstream cylinder vortex shedding" regime.

(a)

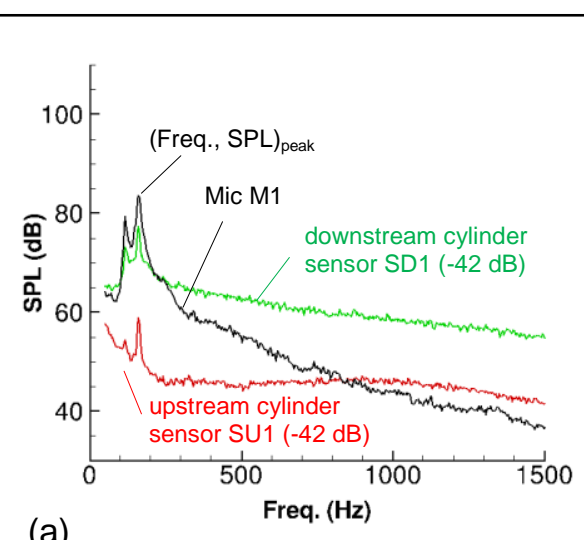

Figure 11. L/D=3.0; Flow Mach number 0.128; upstream cylinder tripped; (a) Noise and surface pressure spectra; (b) Spanwise coherence factor level at the noise spectral peak frequency. 
Finally, the noise, surface pressure spectra and spanwise coherence obtained for L/D of 1.435 and a Mach number of 0.128 are presented in Figure 12(a) and (b). This configuration was found to be the lowest noise configuration tested. These spectra contain small, broad peaks, and the spanwise coherence between surface pressure sensor signals is very weak. The same characteristics were observed at all other flow speeds. PIV measurements ${ }^{17}$ performed for this L/D indicated that this cylinder configuration appears to act as a single bluff body, with the shear layer from the upstream cylinder grazing the sides of the downstream cylinder and possibly re-attaching to the surface of the downstream cylinder. Surface pressure and hot-wire measurements ${ }^{18}$ showed no clear evidence of vortex shedding from either cylinder. In the present study, the noise spectra obtained at each flow speed comprised two small peaks: a first peak (the largest) near $\mathrm{St} \sim 0.14$ and a second (broader) peak near $\mathrm{St} \sim 0.28$. The latter peak was the only one found to be common to both noise and surface pressure spectra (although, as seen in Figure 12(a), it only very weakly appears in the surface pressure spectra of the upstream cylinder). A high St value (such as 0.28) is typically ${ }^{12,19}$ associated with a narrow wake such as that produced behind cylinders acting as a bluff body, while a low St value (such as 0.14 ) is typically ${ }^{19}$ associated with a flow regime where the shear layer from the upstream cylinder intermittently reattaches onto the downstream cylinder. Thus, the results obtained in the present experiment for $\mathrm{L} / \mathrm{D}=1.435$, appear consistent with previous flow visualization studies ${ }^{17,18}$ and with the flow regime approaching that of a bluff body $\left(1^{\text {st }}\right.$ regime listed in Table 1$)$.

In the next section, $\mathrm{St}$ values for $\mathrm{L} / \mathrm{D}=1.435$ (and for all other $\mathrm{L} / \mathrm{D}$ ) were determined using the noise spectra peak SPL frequency.

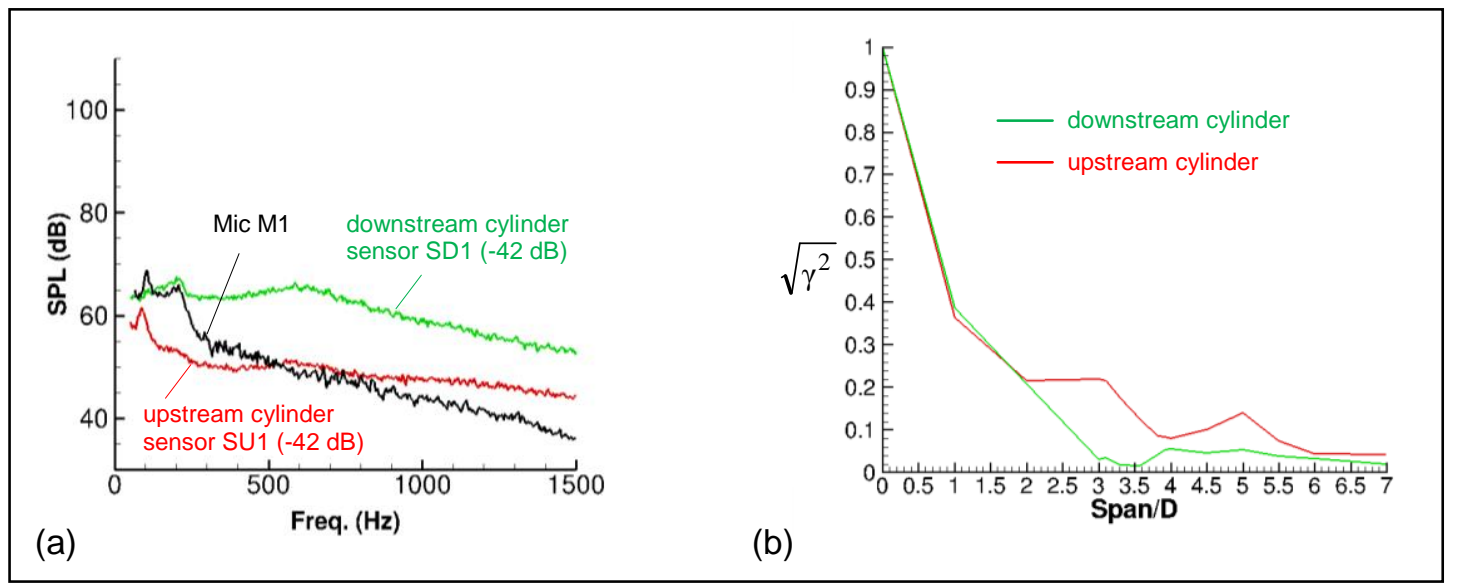

Figure 12. L/D=1.435; Flow Mach number 0.128; upstream cylinder tripped; (a) Noise and surface pressure spectra; (b) Spanwise coherence factor level at the noise spectral peak frequency.

\subsection{Strouhal number and Peak SPL}

The dependence of the Strouhal number (St) and peak SPL on flow velocity is examined in this section. The peak SPL values shown correspond to the peak SPL of the dominant tone in the noise spectra integrated on a $\mathrm{p}^{2}$ basis over 13 frequency bins centered at $\mathrm{f}_{\text {peak }}$.

The variation of St with flow Mach number (for each L/D) is displayed in Figure 13. For the tripped cylinder configurations, $\mathrm{St}$ is seen to increase with $\mathrm{L} / \mathrm{D}$, and while within a given flow regime, St remains constant with increasing Mach number. For the un-tripped cylinder configurations, St is seen to remain constant with increasing Mach number, and to have generally lower values than for the tripped cases.

The variation of the peak SPL with flow Mach number (for each L/D) is displayed in Figure 14. For the tripped cylinder configurations the peak SPL is seen to increase with L/D, and while within a given flow regime, the peak SPL scales with the $6^{\text {th }}$ power of the Mach number (this is shown by the scaled peak SPL levels remaining approximately constant with increasing $\mathrm{M}$ ). For the un-tripped cylinder configurations, the 
peak SPL is seen to also scale with the $6^{\text {th }}$ power of the Mach number, and to have generally higher values than in the tripped cylinder cases.

Note that the switch in flow regime discussed in section 4.2 for $\mathrm{L} / \mathrm{D}=3.0$ is also captured by the drop in St and peak SPL values observed with increasing M.

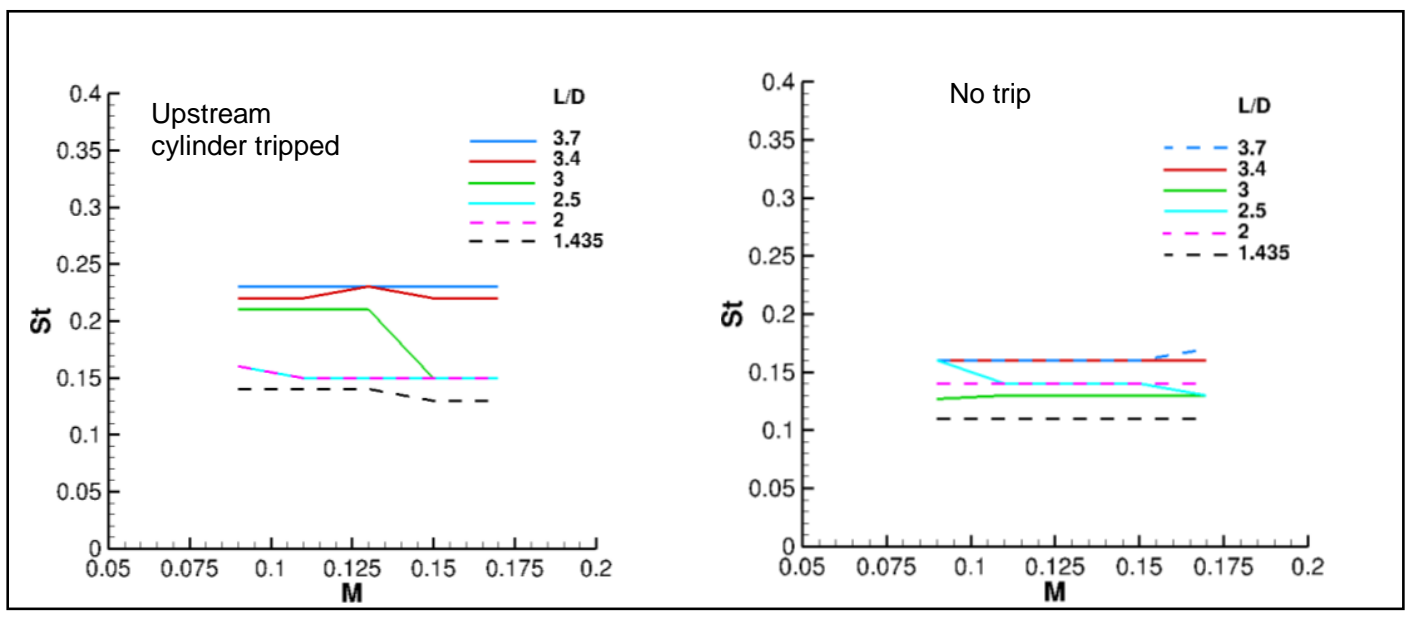

Figure 13. Variation of Strouhal number with flow Mach number.

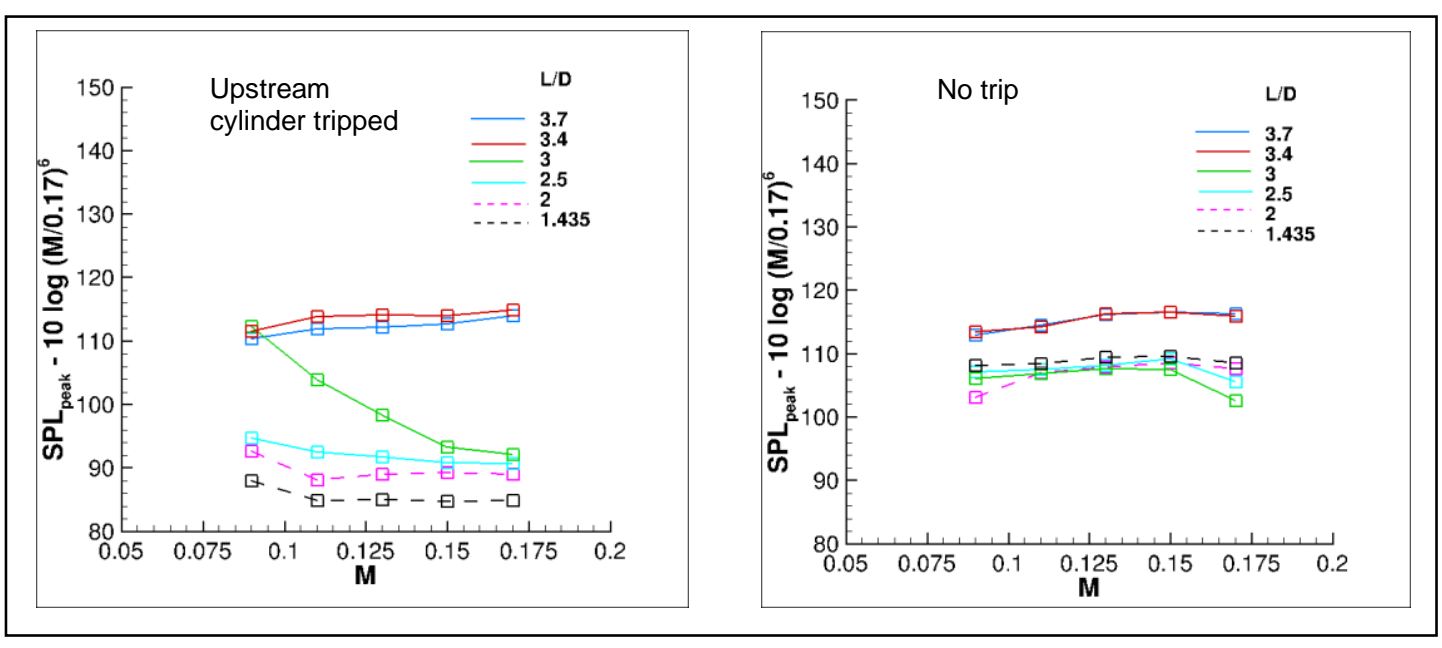

Figure 14. Variation of peak SPL with flow Mach number for each L/D.

To further evaluate the applicability of Eq. (1) for the prediction of the vortex shedding noise radiating from the tandem cylinder configurations tested, the measured peak SPL are scaled with respect to $\left(\mathrm{M}^{6} \mathrm{~L}_{\mathrm{c}}\right.$ $\mathrm{S}_{\mathrm{t}}{ }^{2}$ ). The other parameters in the first term of the right hand side of Eq. (1) are assumed constant. The results obtained for the tripped configurations using the correlation lengths $\left(\mathrm{L}_{c}\right)$ measured either along the upstream or downstream cylinder are shown in Figure 15 (a) and (b), respectively. It is seen that the best data collapse is obtained when using the correlation length from the downstream cylinder for the scaling. In that case, the curves collapse within $8 \mathrm{~dB}$ (versus $20 \mathrm{~dB}$ when using the correlation length from the upstream cylinder). The best data collapse (within 2-3 dB) is achieved for the cylinder configurations 
where the vortex shedding and flow regime is best established (namely, for $\mathrm{L} / \mathrm{D} \geq 2.5$ and except during flow regime transition for $\mathrm{L} / \mathrm{D}=3.0$ ).

When using the correlation length from the upstream cylinder for scaling, the peak SPL values measured for $\mathrm{L} / \mathrm{D}=3.0$ (when $\mathrm{M} \geq 0.15$ ), 2.5 and 2.0 are not collapsing with the rest of the data. This result is not unexpected since the surface pressure spectra and spanwise coherence level obtained for these cylinder configurations (and showed previously) indicate that only the downstream cylinder contributes to the vortex shedding.

For the un-tripped cylinder configurations, the collapse of the data seen in Figure 14 (data scaled with respect to $\mathrm{M}^{6}$ ) was not improved when scaling with respect to $\left(\mathrm{M}^{6} \mathrm{~L}_{\mathrm{c}} \mathrm{S}_{\mathrm{t}}^{2}\right)$, regardless of whether the upstream or downstream cylinder's correlation length was used.

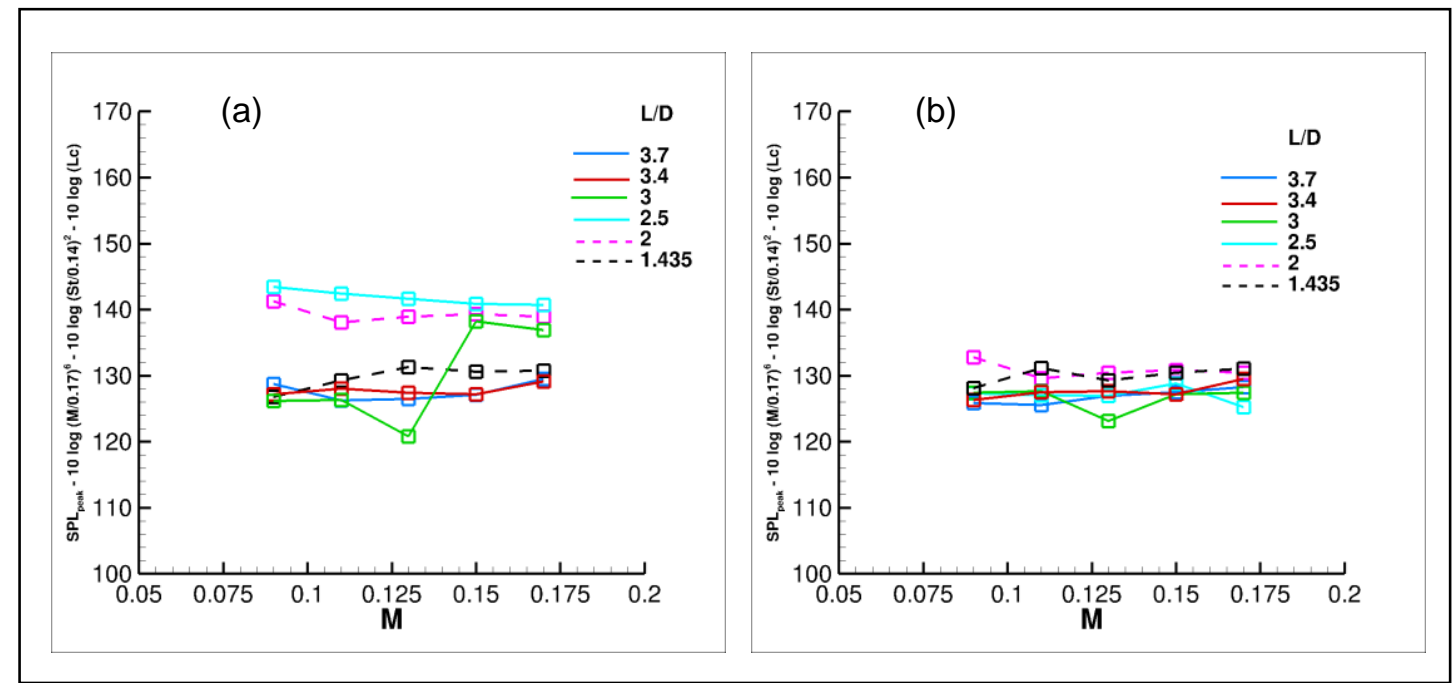

Figure 15. Variation of peak SPL with flow Mach number. Upstream cylinder is tripped. Peak SPL values scaled w.r.t. $\left(\mathrm{M}^{6} \mathrm{St}^{2} \mathrm{Lc}\right)$ using: (a) Lc from upstream cylinder; (b) Lc from downstream cylinder.

\subsection{Noise radiation Directivity}

The directivity measurements were obtained for the $\mathrm{L} / \mathrm{D}=3.7$ and $\mathrm{L} / \mathrm{D}=1.435$ configurations. These results are presented as a function of emission angles in Figures 16 and 17, respectively. The spectral peak levels and emission angles $\Phi_{\mathrm{e}}$ corresponding to each microphone location were corrected to account for shear-layer effects ${ }^{24}$. To calculate the shear layer corrections, a source location at the center of the downstream cylinder, and a straight shear-layer were assumed. The theoretical compact dipole directivity pattern (Eq. (3)) is shown for reference. The theoretical directivity peak levels are normalized to the output obtained at elevation $\phi=90^{\circ}$.

For $\mathrm{L} / \mathrm{D}=3.7$, the measured data is seen to be in good agreement with the theoretical directivity pattern. The slight shift seen between the measured and theoretical directivity patterns may be due to combining radiated noise from each cylinder with differing amplitude and phase at the measurement locations. Similar good agreement between measured and theoretical directivity patterns were also observed in a previous study ${ }^{12}$ for the vortex shedding noise from single cylinder configurations. 


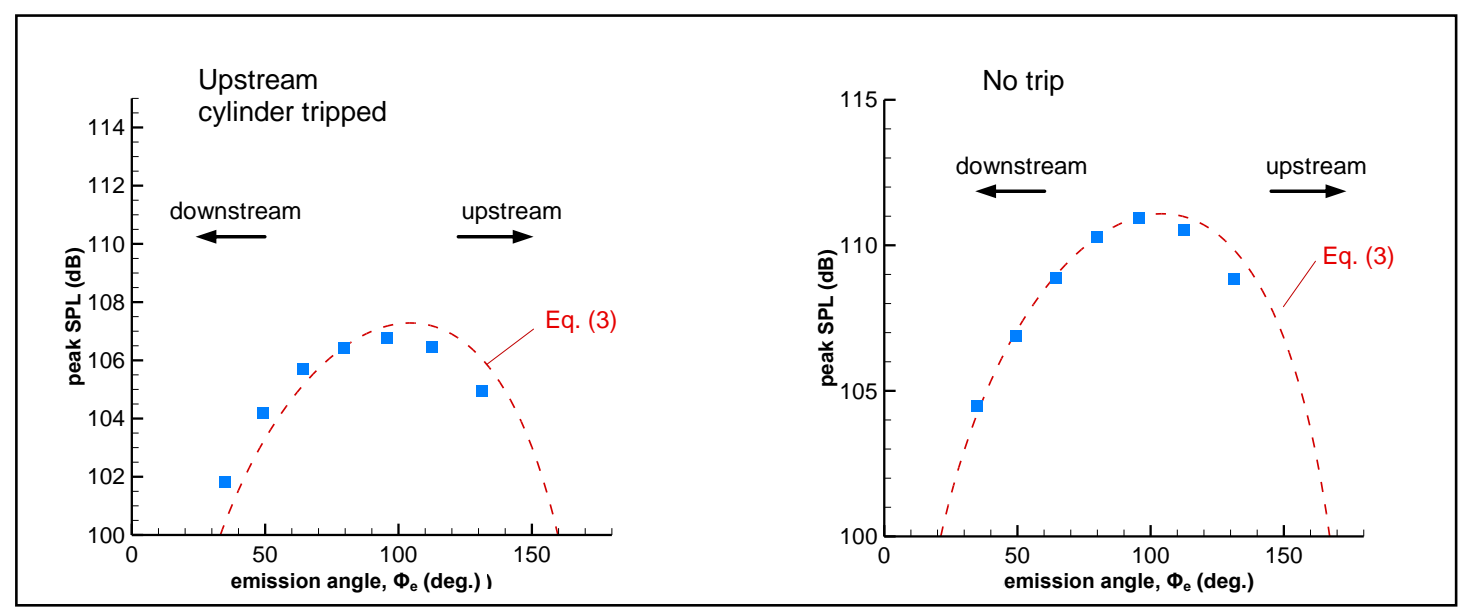

Figure 16. Peak SPL radiation directivity; $L / D=3.7$; flow Mach number 0.128. $\backsim$ : measured data. : theoretical directivity from Eq. (3) with peak levels normalized to the measured data at elevation angle $\phi=90^{\circ}$ (corresponding emission angle $\phi_{\mathrm{e}}=79.7^{\circ}$ ).

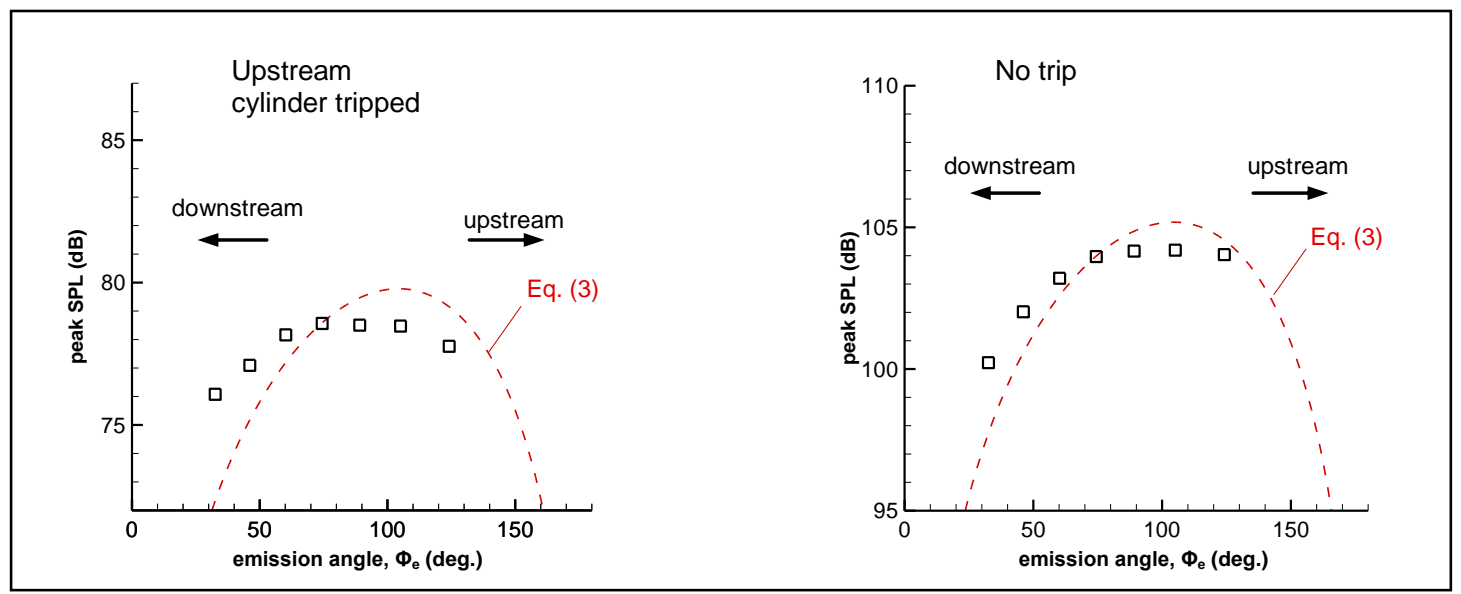

Figure 17. Peak SPL radiation directivity; $L / D=1.435$; flow Mach number 0.128. व: measured data. : theoretical directivity from Eq. (3) with peak levels normalized to the measured data at elevation angle $\phi=90^{\circ}$ (corresponding emission angle $\phi_{\mathrm{e}}=\mathbf{7 4 . 4 ^ { \circ }}$ ).

For $\mathrm{L} / \mathrm{D}=1.435$, with the cylinders un-tripped, the directivity measurements agree relatively well (within $2 \mathrm{~dB}$ ) with the theoretical compact dipole directivity pattern. However, with the tripped cylinder configurations (for which the noise spectrum only displays small broad "tones"), the measured directivity differs by up to $5 \mathrm{~dB}$ from the pattern associated with Eq. (3). No directivity measurements were performed at other $\mathrm{L} / \mathrm{D}$ values.

These results indicate that Eq. (3) best models the radiation directivity of the noise from tandem cylinder configurations where vortex shedding is well established (such as for $\mathrm{L} / \mathrm{D}=3.7$ ). 


\section{Summary}

Detailed noise and surface pressure measurements were performed on tandem cylinder configurations. The model cylinders were tested with and without surface trip. The noise spectra produced when tripping only the upstream cylinder differed little from that produced when both cylinders were tripped. For the smooth cylinder configurations, higher peak and broadband noise levels were observed, and no notable change in flow regime occurred for all L/D and flow speeds. For the tripped cylinder configurations, change in L/D led to different flow regimes, each associated with different noise and unsteady surface pressure spectral characteristics. For all tandem cylinder configurations, St was found to increase with L/D, and (if no regime change) stayed constant with increasing flow speed. Regardless of surface trip, the peak SPL was found to scale with the $6^{\text {th }}$ power of the flow Mach number, and increased with L/D (when the change in $\mathrm{L} / \mathrm{D}$ was associated with a flow regime change). With surface trip, peak SPL were found to best scale with $\left(\mathrm{M}^{6} \mathrm{Lc} \mathrm{St}^{2}\right)$ for the cylinder configurations where the vortex shedding and flow regime is well established. Finally, the noise directivity measured at the peak SPL frequency was found to generally agree with the theoretical directivity for a translating dipole for the tandem cylinder configurations where vortex shedding is prominent.

\section{References}

1. Smith, M. G., Chow, L. C. "Prediction Method for Aerodynamic Noise from Aircraft Landing Gear", AIAA paper 98-2228.

2. Smith, M. G., Chow, L. C. "Validation of a Prediction Model for Aerodynamic Noise from Aircraft Landing Gear", AIAA paper 2002-2581.

3. Souliez, F. J., Long, L. N., Morris, P. J. and Sharma, A. "Landing Gear Aerodynamic Noise Prediction Using Unstructured Grids", AIAA paper 2002-0799.

4. Lockard, D. and Khorrami, M. “Aeroacoustics Analysis of a Simplified Landing Gear", AIAA paper 2003-3111.

5. Guo, Y. “A Statistical Model for Landing Gear Noise Prediction”, AIAA paper 2003-3227.

6. Guo, Y., Yamamoto, K. and Stoker, R. "An Empirical Model for Landing Gear Noise Prediction", AIAA paper 2004-2888.

7. Seror, C., Sagaut, p. and Belanger, A. "A Numerical Aeroacoustic Analysis of a Detailed Landing Gear" AIAA paper 2004-2884.

8. Ravetta, P., Burdisso, R. and Ng, W. "Wind Tunnel Aeroacoustic Measurements of a 26\%-scale 777 Main Landing Gear", AIAA paper 2004-2885.

9. Jaeger, S., Burnside, N., Soderman, P., Horne, W. and James, K. "Microphone Array Assessment of an Isolated, 26\%-Scale, High-Fidelity Landing Gear", AIAA paper 2002-2410.

10. Dobrzynski, W. and Buchholz, H. "Full Scale Noise Testing on Airbus Landing Gears in the German Dutch Wind Tunnel”, AIAA Paper No. 97-1597.

11. Dobrzynski, W., Chow, L., Guion, P., Shiells, D. "Research into Landing Gear Airframe Noise Reduction", AIAA paper 2002-2409. 
12. Hutcheson, F. and Brooks, T. "Noise Radiation from Single and Multiple Rod Configurations", International Journal of Aeroacoustics, Vol.11 (3\&4), 2012.

13. Hutcheson, F., Brooks, T., Stead, D., Becker, L. and Price, J. "Landing Gear Components Noise Study - PIV and Hot-Wire Measurements", NASA/TM-2010-216209.

14. Lockard, D., Khorrami, M., Choudhari, M., Hutcheson, F., Brooks, T., and Stead, D., "Tandem Cylinder Noise Predictions", AIAA Paper 2007-3450.

15. Lockard, D., Choudhari, M., Khorrami, M., Neuhart, D., Hutcheson, F., Brooks, T., and Stead, D., "Aeroacoustic Simulations of Tandem Cylinders with Subcritical Spacing", AIAA Paper 20082862.

16. Khorrami, M., Choudhari, M., Jenkins, L., and McGinley, C., "Unsteady Flowfield around Tandem Cylinders as Prototype for Component Interaction in Airframe Noise”, AIAA Paper 20052866.

17. Jenkins, L., Khorrami, M., Choudhari, M., and McGinley, C., "Characterization of Unsteady Flow Structures around Tandem Cylinders for Component Interaction Studies in Airframe Noise", AIAA Paper 2005-2812.

18. Jenkins, L., Neuhart, D., McGinley, C., Choudhari, M., and Khorrami, "Measurements of Unsteady Wake Interference between Tandem Cylinders", AIAA Paper 2006-3202.

19. Zdravkovich, M. M. "Flow around Circular Cylinders, Volume 2", Oxford University Press, 1997.

20. Schlinker, R., Fink, M., Amiet, R., "Vortex Noise from Nonrotating Cylinders and Airfoils", AIAA paper 76-81, 1976.

21. Fink, M., Schlinker, R., Amiet, R., "Prediction of Rotating Blade Vortex Noise from Noise of NonRotating Blades" NASA CR-2611.

22. Brooks, T., Pope, D., Marcolini, M., “Airfoil Self-Noise and Prediction”, NASA reference publication 1218, July 1989.

23. Hutcheson, F., Brooks, T., "Measurement of Trailing Edge Noise using Directional Array and Coherent Output Power Methods", AIAA paper 2002-2472.

24. Amiet, R., "Refraction of Sound by a Shear-Layer", Journal of Sound and Vibration, Vol. 58 (3), 467482, 1978.

25. Brooks, T., Hodgson, T., "Trailing Edge Noise Prediction from Measured Surface Pressures”, Journal of Sound and Vibration, Vol. 78 (1), 69-117, 1981. 